E D I T O R I A L S

\title{
Treatment of Malaria - A Continuing Challenge
}

\author{
Brian Greenwood, M.D.
}

Twenty years ago, malaria was a neglected disease that was responsible for at least 1 million deaths per year; this high mortality was due in part to reliance on chloroquine, a failing drug, for treatment. ${ }^{1}$ Since then, there has been substantial progress in malaria control, and the number of deaths from malaria has been reduced by about one third. However, the World Health Organization (WHO) estimates that in 2012, malaria caused 627,000 deaths (estimated range, $473,000$ to 789,000$),{ }^{2}$ so there is still much more to be done. An important contributor to recent success in malaria control has been the widespread use of highly effective artemisinin-based combination therapies. Hence, the emergence in Southeast Asia of Plasmodium falciparum parasites that are partially resistant to artemisinin is of great concern..$^{3,4}$

Until recently, the only way to detect artemisinin resistance was by measuring the parasite clearance rate among patients who received artemisinin monotherapy. In this issue of the Journal, Ashley and colleagues ${ }^{5}$ describe their use of this rather cumbersome assay to map the presence of artemisinin-resistant $P$. falciparum parasites across Southeast Asia and at three sites in Africa. Slowly clearing infections (parasite clearance half-life $>5$ hours) were detected across Southeast Asia; the highest prevalence was in western Cambodia, but the parasite clearance half-life was 5 hours or less at one site in India and at three sites in Africa. However, because of the paucity of sampling sites in India and Africa, it cannot be assumed that resistant parasites are not already present in these areas, and more extensive studies, some of which are already under way, are needed to investigate this.
The performance of in vivo assays is demanding, and mapping the prevalence of artemisininresistant parasites is being facilitated by the recent discovery of a molecular marker of artemisinin resistance. ${ }^{6}$ Ariey and colleagues found mutations in the "propeller" domain of the kelch protein gene in a parasite line in which resistance had been induced in the laboratory, and they showed that these mutations were associated with delayed parasite clearance in Cambodia. ${ }^{6}$ This association is further supported in the study by Ashley and colleagues.

What can be done to contain the threat of artemisinin resistance? First, surveillance for resistant parasites in areas where they have not yet been detected must be enhanced and sustained. Second, artemisinin monotherapy must be strongly discouraged. Although most national malariacontrol programs have heeded the advice of the WHO to prohibit the use of artemisinin monotherapy, ${ }^{7}$ it is still being used widely in the private sector. What could be done if a new focus of artemisinin-resistant parasites is detected outside Southeast Asia? If resistant parasites are confined to a geographically restricted area, a strong case can be made for a "blitz" campaign aimed at eliminating the resistant parasites before they can spread. This campaign would involve the use of all available means, including enhanced vector control, mass drug administration, and perhaps vaccination (the RTS,S/AS01 vaccine may become available in 2016). Whether or not such a campaign would succeed is uncertain, and thus clinicians who work in areas where malaria is endemic must be prepared for an era in which artemisinin-based combination therapies are no longer effective. 
Chloroquine was such an effective drug that little was done to prepare for its failure. Fortunately, scientists in academia and pharmaceutical companies have learned from this lack of preparation, and despite the current success of artemisinin-based combination therapies, an active program of development of antimalarial drugs has been sustained during the past two decades. This program has been led by the Medicines for Malaria Venture ${ }^{8}$ with strong support from academia and the pharmaceutical industry. Consequently, several promising new antimalarial drugs are in the pipeline. The results of an early trial of one of these new compounds are described by White and colleagues ${ }^{9}$ in this issue of the Journal. This drug (KAE609), a spiroindolone developed by Novartis, has a novel mechanism of action targeting a parasite plasma membrane $\mathrm{Na}^{+}$-ATPase, and it is effective against both asexual-stage and sexual-stage parasites. ${ }^{10}$ Three days of treatment (at a dose of $30 \mathrm{mg}$ per day) led to very rapid parasite clearance in 11 patients with $P$. falciparum malaria (including 5 patients infected with parasites carrying the kelch protein resistance mutation) and 10 patients with P. vivax malaria. The main side effects were nausea and vomiting. Spiroindolone-resistant mutations can be induced in vitro, so if KAE609 is to be used widely, it will need to be combined with another antimalarial compound with a different mechanism of action.

The emergence of artemisinin-resistant parasites is a major threat to further advances in malaria control. Every effort needs to be made to contain their spread while at the same time pushing forward with the development of effective alternative treatments that are almost certainly going to be needed in the future.

Disclosure forms provided by the author are available with the full text of this article at NEJM.org.

From the London School of Hygiene and Tropical Medicine, London.

1. Trape JF, Pison G, Preziosi MP, et al. Impact of chloroquine resistance on malaria mortality. C R Acad Sci III 1998;321:68997.

2. World malaria report 2013. Geneva: World Health Organization, 2013.

3. Dondorp AM, Nosten F, Yi P, et al. Artemisinin resistance in Plasmodium falciparum malaria. N Engl J Med 2009;361:455-67. [Erratum, N Engl J Med 2009;361:1714.]

4. Phyo AP, Nkhoma S, Stepniewska K, et al. Emergence of artemisinin-resistant malaria on the western border of Thailand: a longitudinal study. Lancet 2012;379:1960-6.

5. Ashley EA, Dhorda M, Fairhurst RM, et al. Spread of artemisinin resistance in Plasmodium falciparum malaria. $\mathrm{N}$ Engl $\mathrm{J}$ Med 2014;371:411-23.

6. Ariey F, Witkowski B, Amaratunga C, et al. A molecular marker of artemisinin-resistant Plasmodium falciparum malaria. Nature 2014;505:50-5.

7. Global plan for artemisinin resistance containment (GPARC). Geneva: World Health Organization, 2011.

8. Medicines for Malaria Venture home page (http://www.mmv .org).

9. White NJ, Pukrittayakamee S, Phyo AP, et al. Spiroindolone KAE609 for falciparum and vivax malaria. N Engl J Med 2014; 371:403-10.

10. Spillman NJ, Allen RJW, McNamara CW, et al. $\mathrm{Na}^{+}$regulation in the malaria parasite Plasmodium falciparum involves the cation ATPase PfATP4 and is a target of the spiroindolone antimalarials. Cell Host Microbe 2013;13:227-37.

DOI: 10.1056/NEJMe1407026

Copyright (@) 2014 Massachusetts Medical Society. at NEJMjobs.org for more information. 